\title{
OPEN A scaffolded approach to unearth potential antibacterial components from epicarp of Malaysian Nephelium lappaceum L.
}

\author{
Ali Asghar ${ }^{1}$, Yong Chiang Tan ${ }^{1}$, Mohammad Zahoor ${ }^{2}$, Syafiq Asnawi Zainal Abidin ${ }^{3}$, \\ Yoon-Yen Yow ${ }^{1}$, Ezzat Khan ${ }^{4} \&$ Chandrajit Lahiri ${ }^{1 凶}$
}

The emergence and spread of antimicrobial resistance have been of serious concern to human health and the management of bacterial infectious diseases. Effective treatment of these diseases requires the development of novel therapeutics, preferably free of side effects. In this regard, natural products are frequently conceived to be potential alternative sources for novel antibacterial compounds. Herein, we have evaluated the antibacterial activity of the epicarp extracts of the Malaysian cultivar of yellow rambutan fruit (Nephelium lappaceum L.) against six pathogens namely, Bacillus subtilis, methicillin-resistant Staphylococcus aureus (MRSA), Streptococcus pyogenes, Pseudomonas aeruginosa, Klebsiella pneumoniae and Salmonella enterica. Among a series of solvent extracts, fractions of ethyl acetate and acetone have revealed significant activity towards all tested strains. Chemical profiling of these fractions, via HPLC, LC-MS and GC-MS, has generated a library of potentially bioactive compounds. Downstream virtual screening, pharmacological prediction, and receptor-ligand molecular dynamics simulation have eventually unveiled novel potential antibacterial compounds, which can be extracted for medicinal use. We report compounds like catechin, eplerenone and oritin-4-beta-ol to be computationally inhibiting the ATP-binding domain of the chaperone, DnaK of $P$. aeruginosa and MRSA. Thus, our work follows the objective to propose new antimicrobials capable of perforating the barrier of resistance posed by both the gram positives and the negatives.

Antimicrobial resistance (AMR) has been projected as one of the serious global concerns with issues in the management of infectious diseases caused by Multidrug-Resistant (MDR) bacterial pathogens ${ }^{1}$. These pathogens have been reported to cause 700,000 deaths each year and is estimated to cross over 10 million by $2050^{2}$. Such alarming rise can be attributed mostly to the prevalent misappropriation of antibiotics in human healthcare systems $^{3}$. This is delineated by the overuse and poor appropriate prescriptions coupled with the lack of new drug development, which have reduced the efficiency of antibiotics as the resistant strains rapidly increases ${ }^{4}$. This necessitates the urgency for unearthing alternate natural therapeutics focused on natural products to be exploited as repertoires of natural bioactive compounds, preferably devoid of side effects and hence, the potential for future drug development.

Natural products, frequently discovered with potent antimicrobial potential, have been used as alternatives with the hope of eliminating the use of synthetic antibiotics, for a long time ${ }^{5}$. Besides serving a wide range of secondary plant metabolites, for instance, alkaloids, tannins, flavonoids and phenolic compounds, with remarkable antimicrobial effects ${ }^{6}$, many plant products have been reported for their antimicrobial potentials, namely, citrus peel ${ }^{7}$, grape seed ${ }^{8}$, cranberry pomace ${ }^{9}$, pomegranate pee ${ }^{10}$ and passion fruit seed ${ }^{11}$. In this regard, the fruit rambutan (Nephelium lappaceum L.) has gained attention due to its vast range of bioactive constituents including, but not limited to, vitamin C, vitamin E, carotenes, xanthophylls, tannins and phenolic compounds like geraniin, ellagic acid, quercetin, corilagin and rutin ${ }^{12}$.

While the broad range of biological activities of rambutan includes anticancer, antiviral, antidiabetic and anti-hypercholesterolemic properties ${ }^{13,14}$, few researchers have even reported its in vitro antibacterial activities.

\footnotetext{
${ }^{1}$ Department of Biological Sciences, Sunway University, Petaling Jaya, Malaysia. ${ }^{2}$ Department of Biochemistry, University of Malakand, Chakdara, Pakistan. ${ }^{3}$ Jeffrey Cheah School of Medicine and Health Science, Monash University Malaysia, Petaling Jaya, Malaysia. "Department of Chemistry, University of Bahrain, Sakhir, Bahrain. ${ }^{\varpi}$ email: chandrajitl@sunway.edu.my
} 


\begin{tabular}{|l|l|l|l|l|l|l|l|l|l|l|}
\hline \multicolumn{2}{|l|}{ Zones of inhibition $(\mathbf{m m})$} \\
\hline Species & PC & SC & CF & EA & AC & ET & MT & WT & EA(D) & AC(D) \\
\hline B. subtilis & $30.00 \pm 0.70$ & - & - & - & - & - & - & - & $8.80 \pm 0.65$ & $9.39 \pm 0.43$ \\
\hline MRSA & & - & - & - & - & - & - & - & $8.58 \pm 0.29$ & - \\
\hline S. pyogenes & & - & - & - & - & - & - & - & - & $8.35 \pm 0.45$ \\
\hline P. aeruginosa & & - & - & - & - & - & - & - & - & $8.00 \pm 0.01$ \\
\hline K. pneumoniae & & - & - & - & - & - & - & - & - & - \\
\hline S. enterica & & - & - & - & - & - & - & - & $6.60 \pm 0.28$ & - \\
\hline
\end{tabular}

Table 1. Antibacterial activity of the N. lappaceum sequential and direct crude extracts via disc diffusion. “_" no activity, PC: positive control (Gentamicin $10 \mu \mathrm{g}$ ), SC: solvent control (DMSO < 1\%), CF: chloroform, EA: ethyl acetate, AC: acetone, ET: ethanol, MT: methanol, WT: water, all these are sequential. EA(D): ethyl acetate direct; $\mathrm{AC}(\mathrm{D})$ : acetone direct. The data is expressed as the mean \pm standard error of two independent experiments performed in technical triplicates.

For instance, Malini \& Maheshkumar ${ }^{15}$ have disclosed significant antibacterial activity of rambutan fruit sap extracts towards Pseudomonas aeruginosa while Bhat and Al-daihan ${ }^{16}$ revealed antibacterial activities of its seeds extracts against Staphylococcus aureus, Streptococcus pyogenes, Bacillus subtilis, Escherichia coli and P. aeruginosa. Moreover, the antibacterial potential of rambutan peel extracts have also been reported against Vibrio cholerae, Enterococcus faecalis, S. aureus and Staphylococcus epidermidis ${ }^{17}$. Furthermore, Sekar et al. ${ }^{18}$ comparatively evaluated the antibacterial potency of red and yellow rambutan fruit peels against $S$. aureus and $S$. pyogenes, to reveal better efficacy of the latter extracts against the tested pathogens. However, a deeper exploration of the potential extracts to unveil new antibacterial compounds have hardly been focused.

In this study, thus, we have delineated a stepwise approach of determining the efficacy of the crude extracts of the epicarp of yellow Malaysian rambutan against clinically important MDR bacterial pathogens e.g., $B$. subtilis, methicillin-resistant S. aureus (MRSA), S. pyogenes, P. aeruginosa, K. pneumoniae and S. enterica. To this end, through HPLC, LC-MS and GC-MS analyses, we have prepared chemical profiling of the ethyl acetate and acetone fractions of the crude extracts with promising antibacterial activities. This revealed their potential chemical determinants which were screened virtually to pharmacologically unveil novel bioactive compounds in parallel to molecular dynamics simulation. This enabled us to elect compounds like catechin, eplerenone and oritin-4-beta-ol, which computationally inhibit the important chaperone protein, DnaK of P. aeruginosa and MRSA. Essentially, this report serves to unveil these compounds as novel alternatives to cope with the multidrugresistant gram-positive and -negative pathogens.

\section{Results}

Variable antibacterial activity of crude extracts by disc diffusion. We have preliminarily screened the antibacterial activities of the yellow-variety Malaysian Rambutan epicarp crude extracts through disc diffusion assay. We have only used freshly prepared solutions of crude extracts for all the tested pathogens (TP). During such trials, the solvent control (SC), DMSO, did not exercise antibacterial activity against the TP, as manifested by a no inhibition zone. Moreover, none of the sequential extracts exhibited activity against the TP (Table 1), while in the case of direct extracts, the picture was different. Extracts of ethyl acetate (EA) displayed visible activity against MRSA, B. subtilis and S. enterica but did not show activity against the rest of the TP (RTP). Again, acetone (AC) extracts exhibited markedly prominent activity against $B$. subtilis along with visible activity against $S$. pyogenes and P. aeruginosa, which was not observed among the RTP (Table S1).

Antibacterial screening of crude extracts via Broth dilution. Next, we have used the broth dilution method for evaluating the antibacterial potential of yellow fruit epicarp crude extracts and calculating the viability percentage of every TP (Table 2). This, in turn, helped us to illustrate the percentages of antibacterial potential of EA and AC fractions from the sequential \& direct extracts, against the TP, at a concentration of $250 \mu \mathrm{g} / \mathrm{ml}$. For the sequential extracts, the fraction from AC exhibited the highest percentage (90) of antibacterial activity against $P$. aeruginosa followed by $71 \%$ against MRSA while that from EA showed a $59 \%$ effect against S. pyogenes. No significant results were observed either against the RTP (Fig. S3A-F) or for the remaining solvent extracts namely, chloroform (CF), ethanol (ET), methanol (MT) and aqueous (water, WT) extracts against all TP (Table S2). In the case of direct extracts with the six solvents used, fractions of EA exhibited inhibition of $80 \%$ against $B$. subtilis along with 60,62, 73 and $72 \%$ for MRSA, P. aeruginosa, S. enterica and K. pneumonia, respectively, without any positive results against $S$. pyogenes. Notably, all tested pathogens were inhibited by AC fractions and the percentage of antibacterial effects were 75, 90, 70, 70, 60 and 75, respectively for MRSA, $B$. subtilis, S. pyogenes, P. aeruginosa, S. enterica and K. pneumonia (Fig. S1A-D).

The results of EA and AC fractions portrayed notable antibacterial efficiency. We have, thus, utilized them for the identification of bioactive compounds via HPLC, LC-MS and GC-MS analyses.

Revelation of antioxidants from crude extracts using HPLC-UV. At first, we have conducted HPLC-UV, for preliminary identification of the basic antioxidants present in the Malaysian yellow-rambutan epicarp extracts. All compounds with known antioxidant capacities were identified in comparison with standard 


\begin{tabular}{|l|l|l|c|l|l|l|}
\hline \% Inhibition of all tested pathogen \\
\hline Microorganisms & S.C & P.C & EA(S) & EA(D) & AC(S) & AC(D) \\
\hline B. subtilis & $02 \pm 0.00$ & $100 \pm 0.00$ & - & $80 \pm 1.98$ & - & $90 \pm 1.42$ \\
\hline MRSA & 02 & 100 & - & $60 \pm 1.49$ & $71 \pm 1.91$ & $75 \pm 2.04$ \\
\hline S. pyogenes & 02 & 100 & $59 \pm 1.33$ & - & - & $70 \pm 1.40$ \\
\hline P. aeruginosa & 02 & 100 & - & $62 \pm 1.83$ & $90 \pm 1.58$ & $70 \pm 2.02$ \\
\hline K. pneumonia & 02 & 100 & - & $72 \pm 1.79$ & - & $75 \pm 2.61$ \\
\hline S. enterica & 02 & 100 & - & $73 \pm 1.84$ & - & $60 \pm 2.74$ \\
\hline
\end{tabular}

Table 2. Screening of antibacterial effect of sequential and direct extracts by broth dilution method. EA(S): ethyl acetate sequential; $\mathrm{AC}(\mathrm{S})$ : acetone sequential; $\mathrm{EA}(\mathrm{D})$ : ethyl acetate direct; $\mathrm{AC}(\mathrm{D})$ : acetone direct; S.C: solvent control: DMSO (<1\%), P.C: positive control (Gentamicin $10 \mu \mathrm{g})$; - : Low activity (less than 50\%). The data is expressed as the mean \pm standard error of two independent experiments performed in technical triplicates.

\begin{tabular}{|l|l|l|c|}
\hline Sample extract & RT (min) & Identified compounds & Peak area \\
\hline \multirow{4}{*}{ EA (S) } & 12.9 & Malic acid & 40.441 \\
\cline { 2 - 4 } & 4.8 & Vitamin C & 1717.201 \\
\cline { 2 - 4 } & 6.2 & Chlorogenic acid & 190.156 \\
\hline \multirow{5}{*}{ AC (S) } & 2.9 & Malic acid & 4.938 \\
\cline { 2 - 4 } & 4.8 & Vitamin C & 1226.316 \\
\cline { 2 - 4 } & 6.2 & Chlorogenic acid & 134.587 \\
\cline { 2 - 4 } & 8.8 & Epigallocatechin gallate & 205.717 \\
\cline { 2 - 4 } & 10.7 & Quercetin & 85.112 \\
\cline { 2 - 4 } & 20.7 & Catechin hydrate & 44.169 \\
\hline
\end{tabular}

Table 3. Identified compounds in N. lappaceum ethyl acetate and acetone sequential fractions using HPLCUV.

phenolic compounds. The identified compounds and their quantification, along with their specific peak position and retention time (Rt) in the chromatogram, are shown in Table 3 and Fig. S2. We identified only three compounds in the EA extract namely, malic acid, vitamin $\mathrm{C}$ and chlorogenic acid along with three more in the $\mathrm{AC}$ extract. These are epigallocatechin gallate, catechin hydrate and quercetin.

EA (S): Ethyl acetate sequential fraction; AC (S): Acetone sequential fraction.

Exploration of other chemical determinants through Liquid Chromatography-Mass Spectrometry (LC-MS) Analysis. For a fast, mass-directed exploration of the compounds possibly present in the rambutan epicarp, we have subjected the EA and AC sequential crude extracts to LC-MS analysis (Figs. S3 \& S4). Our analysis revealed the presence of 54 and 44 compounds, respectively, in the above-mentioned EA and AC extracts (Tables S3 \& S4). We have matched them with the identity of known molecules on the Metlin database, keeping a threshold of Molecular Formula Generator (MFG) scores above $86 \%$ along with a $\pm 2 \%$ difference. We further explored the compounds above the mentioned cut-off for their biological activities and carried forward for virtual screening. Notably, 31 compounds from both sets of EA and AC extracts have not been reported to date with any antibacterial activities (Tables S3 \& S4).

Identification of volatile constituents by Gas chromatography-mass spectrometry (GCMS). Hereafter, to identify any volatile organic compounds, present in the EA and AC extracts of Malaysian yellow-variety N. lappaceum epicarp, we have exposed them for GC-MS analysis (Fig. S5a-d). Most of the compounds from EA and AC fractions, extracted directly, have been reported with antibacterial activities (Table 4). On the contrary, the compounds of these fractions from sequential extraction have not been reported for any such activity. The chromatogram of these compounds showed mentionable area \% scores (above $0.5 \%$ ) for 3-Methyl1,2-diazirine (compound 1) and Card-20(22)-enolide, 3-[(6-deoxy-3,4-O-methylenehexopyranos-2-ulos-1-yl) oxy]-5,11/14-trihydroxy-12 -oxo-, (3-beta, 5-alpha, 11-alpha) (compound 2) in the EA extract while, the AC extract showed the presence of Silane, [[(3alpha,5beta,20S)-pregn-11-ene-3,11,17,20-tetrayl] tetrakis(oxy)] tetrakis [trimethyl] and 2,2-Bis[4-[(4,6-dichloro-1,3,5-triazin-2-yl) oxy] phenyl]-1,1,1,3,3,3-hexafluoropropane (Table 4). Of these, compound 2 is known as Eplerenone (Fig. 1) and was found to be an important one in the upcoming analyses.

Short listing of antibacterial compounds via virtual screening and pharmacokinetics. We have inspected the quality of DnaK protein homology models, all of which had good steric properties (Fig. S6). Moreover, we have validated the method for virtual screening via the redocking approach, with minimal RMSD 


\begin{tabular}{|c|c|c|c|c|c|c|}
\hline No & Extracts & Identified compounds & Molecular Formula & R.T. (Min) & Area $\%$ & Antibacterial activity repor \\
\hline 1 & \multirow[b]{2}{*}{ EA (S) } & 3-Methyl-1,2-diazirine & $\mathrm{C}_{2} \mathrm{H}_{4} \mathrm{~N}_{2}$ & 3.02 & 0.776 & Not reported \\
\hline 2 & & $\begin{array}{l}\text { Card-20(22)-enolide, 3-[(6-deoxy-3,4-O-methylenehexopyranos-2-ulos-l-yl) } \\
\text { oxy]-5,11/14-trihydroxy-12 -oxo-, (3- beta.,5-alpha., 11-alpha.) }\end{array}$ & $\mathrm{C}_{30} \mathrm{H}_{40} \mathrm{O}_{11}$ & 3.00 & 3.073 & Not reported \\
\hline 3 & \multirow{2}{*}{$\mathrm{AC}(\mathrm{S})$} & $\begin{array}{l}\text { Silane, [[(3alpha,5beta,20S)-pregn-11-ene-3,11,17,20-tetrayl] tetrakis(oxy)] } \\
\text { tetrakis [trimethyl] }\end{array}$ & $\mathrm{C}_{33} \mathrm{H}_{66} \mathrm{O}_{4} \mathrm{Si}_{4}$ & 41.85 & 5.257 & Not reported \\
\hline 4 & & $\begin{array}{l}\text { 2,2-Bis[4-[(4,6-dichloro-1,3,5-triazin-2-yl) oxy] phenyl]-1,1,1,3,3,3-hexafluor- } \\
\text { opropane }\end{array}$ & $\mathrm{C}_{21} \mathrm{H}_{8} \mathrm{Cl}_{4} \mathrm{~F}_{6} \mathrm{~N}_{6} \mathrm{O}_{2}$ & 3.00 & 4.241 & Not reported \\
\hline 1 & \multirow{8}{*}{ EA (D) } & Phenol, 2,4-bis(1,1-dimethylethyl) & $\mathrm{C}_{14} \mathrm{H}_{22} \mathrm{O}$ & 10.74 & 38.698 & 19 \\
\hline 2 & & Curlone & $\mathrm{C}_{15} \mathrm{H}_{22} \mathrm{O}$ & 14.44 & 4.233 & 20 \\
\hline 3 & & Ar-tumerone & $\mathrm{C}_{15} \mathrm{H}_{20} \mathrm{O}$ & 18.65 & 0.857 & 21 \\
\hline 4 & & Stigmasterol & $\mathrm{C}_{29} \mathrm{H}_{48} \mathrm{O}$ & 17.87 & 1.66 & 22 \\
\hline 5 & & n-Hexadecanoic acid & $\mathrm{C}_{16} \mathrm{H}_{32} \mathrm{O}_{2}$ & 48.78 & 3.2 & 23 \\
\hline 6 & & 3,7,11-Tridecatrienenitrile, 4,8,12-trimethyl & $\mathrm{C}_{16} \mathrm{H}_{25} \mathrm{~N}$ & 23.94 & 2.567 & Not reported \\
\hline 7 & & 2-Methoxy-1,3-dioxolane & $\mathrm{C}_{4} \mathrm{H}_{8} \mathrm{O}_{3}$ & 39.4 & 0.563 & Not reported \\
\hline 8 & & $\begin{array}{l}\text { 5H-Cyclopropa (3,4) benz(1,2-e) azulen-5-one, 1,1a-à,1b-á,4,4a,7a-à,7b,8,9,9a- } \\
\text { decahydro-7b-à,9-á,9a-à-trihydroxy-3-hydroxymethyl-1,1,6,8-à-tetramethyl- } \\
\text { 4a-methoxy-, 9,9a-didecanoate }\end{array}$ & $\mathrm{C}_{41} \mathrm{H}_{66} \mathrm{O}_{8}$ & 58.69 & 2.34 & Not reported \\
\hline 1 & \multirow{6}{*}{$\mathrm{AC}(\mathrm{D})$} & Phenol, 2-methoxy-3-(2-propenyl) & $\mathrm{C}_{10} \mathrm{H}_{12} \mathrm{O}_{2}$ & 10.73 & 0.678 & Not reported \\
\hline 2 & & Phenol, 2,4-bis(1,1-dimethylethyl) & $\mathrm{C}_{14} \mathrm{H}_{22} \mathrm{O}$ & 14.44 & 0.912 & 19 \\
\hline 3 & & Alpha-Tocopherol & $\mathrm{C}_{31} \mathrm{H}_{52} \mathrm{O}_{3}$ & 44.65 & 1.612 & 24 \\
\hline 4 & & Curlone & $\mathrm{C}_{15} \mathrm{H}_{22} \mathrm{O}$ & 18.65 & 1.156 & 20 \\
\hline 5 & & 1,3-Dioxolane, 2-pentadecyl & $\mathrm{C}_{21} \mathrm{H}_{40} \mathrm{O}_{4}$ & 57.36 & 3.06 & Not reported \\
\hline 6 & & Ar-tumerone & $\mathrm{C}_{15} \mathrm{H}_{20} \mathrm{O}$ & 17.87 & 4.081 & 21 \\
\hline
\end{tabular}

Table 4. Compounds existing in N. lappaceum ethyl acetate and acetone (sequential \& direct) extract identified by GC-MS analysis. EA(S): Ethyl acetate sequential; AC(S): Acetone Sequential; EA(D): Ethyl acetate direct; $\mathrm{AC}(\mathrm{D})$ : Acetone direct.<smiles>Oc1cc(O)c2c(c1)OC(c1ccc(O)c(O)c1)C(O)C2</smiles>

Catechin<smiles>Oc1ccc(C2Oc3c(ccc(O)c3O)C(O)C2O)cc1</smiles>

Oritin-4-beta-ol

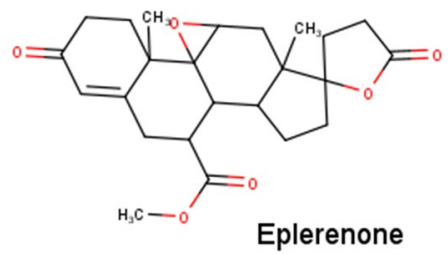

Figure 1. 2D Chemical Structures of Catechin, Oritin-4-beta-ol, and Eplerenone generated using MarvinSketch version 20.16 (http://www.chemaxon.com/ $/)^{31}$.

values (1.715 $\AA$ for 4B9Q and $1.427 \AA$ for 4JNE) between reference and docked poses (Fig. S8). Upon virtual screening of the 91 chemical compounds obtained via chromatographic analyses, we considered 41 of them, with a binding energy of less than $-7 \mathrm{kcal} / \mathrm{mol}$, as good binders (Table S5; Figs. 1, 2A). Among these, we have chosen potential drug candidates based on their predicted pharmacokinetic properties. For example, we prioritized good gastrointestinal (GI) absorption, bad BBB permeability, and non-P-glycoprotein (PGP) substrates for absorption properties. For metabolism, we prioritized non-cytochrome P450 inhibitors. Besides, we avoided violations of drug-likeness rules. To cater to the need, we considered five druggability rules, namely, the Lipinski, Ghose, Veber, Egan, and Muegge rules ${ }^{25-29}$. Lastly, we also prioritized higher bioavailability scores. The Abbot Bioavailability Score utilized herein was to predict chances of drug bioavailability to be more than $10 \%$ upon oral intake ${ }^{30}$.

Among the virtually screened compounds, we found that catechin $(C)$, eplerenone (E) and oritin-4-beta-ol (O) stood out to be good binders with their average binding energies being $-8.205,-7.980$ and $-7.190 \mathrm{kcal} /$ 
A.

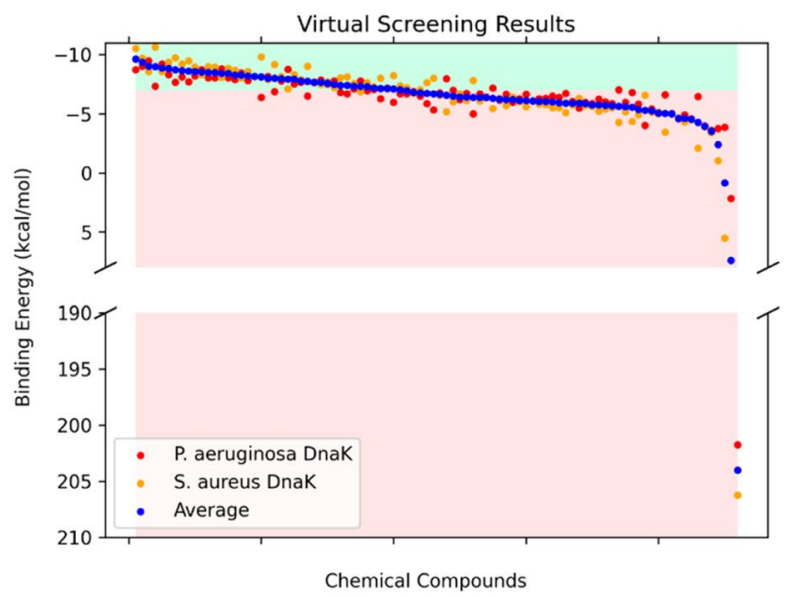

C.
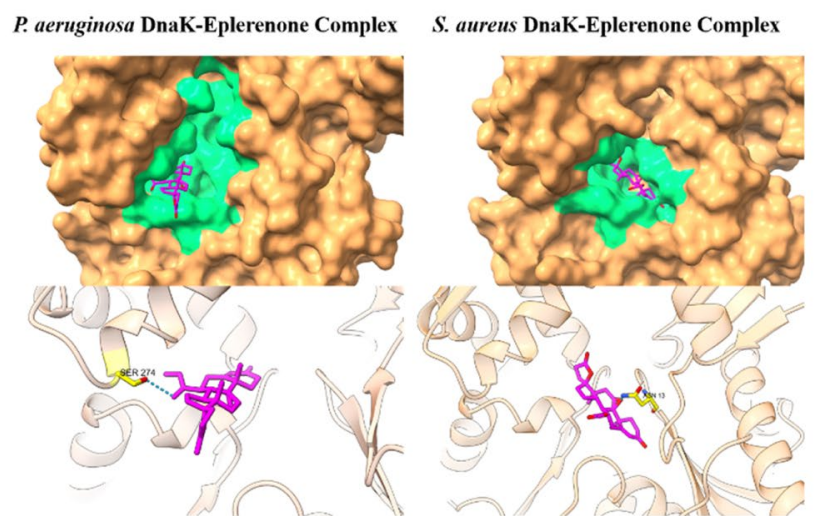

B.

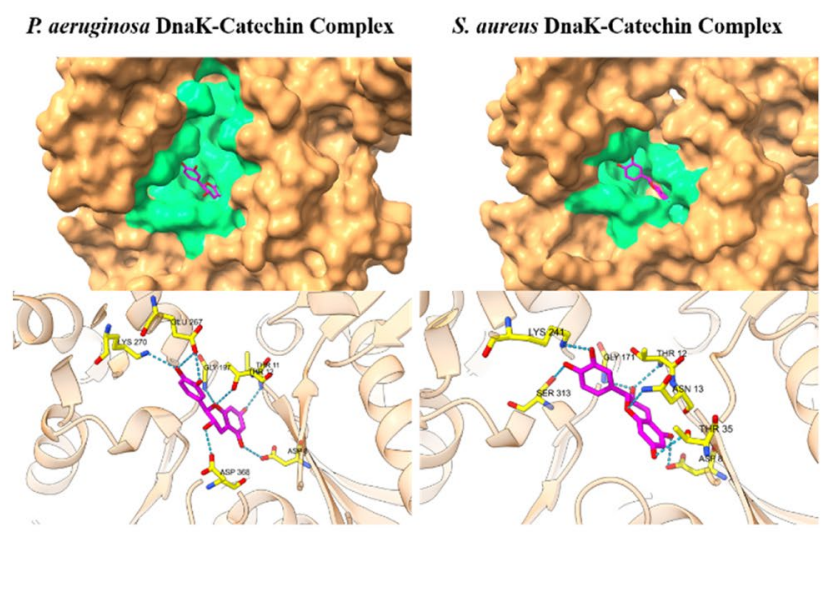

D.

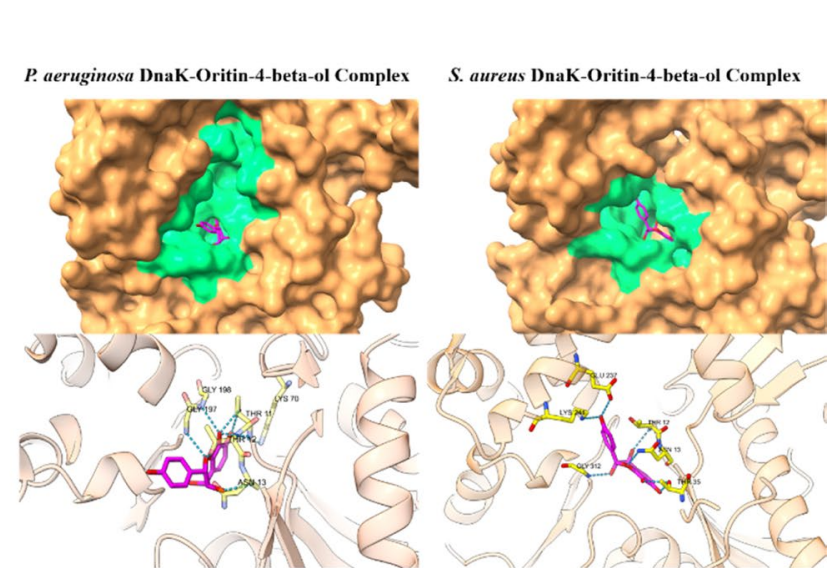

\section{E. ACTIVE RESIDUES}

\begin{tabular}{llcl}
\hline Chemicals & DnaK & Hbonds & Active Residues \\
\hline Catechin & P. aeruginosa & 8 & ASP 8, THR 11, THR 12, GLY 197, GLU 267, LYS 270, ASP 368 \\
& S. aureus & 7 & ASP 8, THR 12, ASN 13, THR 35, GLY 171, LYS 241, SER 313 \\
\hline Eplerenone & P. aeruginosa & 1 & SER 274 \\
& S. aureus & 1 & ASN 13 \\
\hline Oritin-4-beta-ol & P. aeruginosa & 9 & THR 11, THR 12, ASN 13, LYS 70, GLY 197, GLY 198 \\
& S. aureus & 9 & THR 12, ASN 13, THR 35, GLU 237, LYS 241, GLY 312 \\
\hline
\end{tabular}

Note. Hbonds refers to number of hydrogen bonds between receptor and ligand.

Figure 2. Results of virtual screening targeting P. aeruginosa and S. aureus DnaK proteins. (A) Distribution of binding energies plotted via Matplotlib ${ }^{32}$ against screened compounds. Top binders with good pharmacological properties, namely (B) Catechin, (C) Eplerenone, and (D) Oritin-4-beta-ol, generated using UCSF ChimeraX ${ }^{33}$. P2Rank predicted binding pockets were colored in green for visualization of ligands (magenta) binding to the receptor DnaK proteins (brown), with active sites (yellow) labelled. (E) Intermolecular hydrogen bonds tabulated with active residues listed. 
A

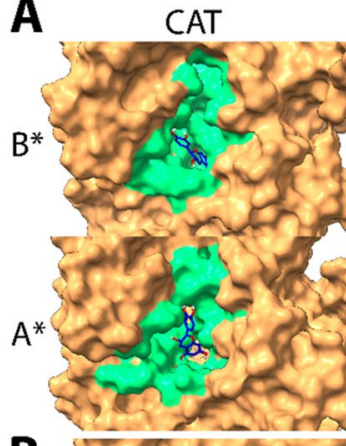

B

$\mathrm{B}^{*}$

$A^{*}$

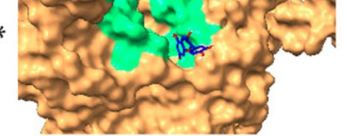

EPL

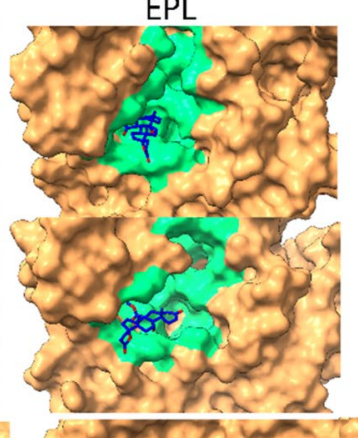

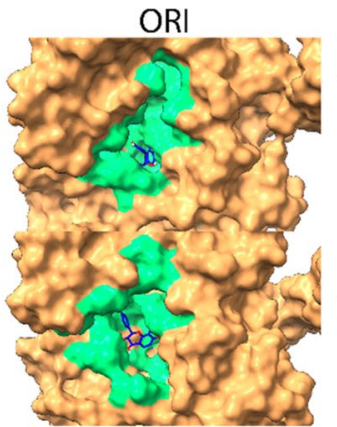

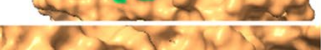

C

\begin{tabular}{clrc}
\hline Ligand & Bacteria & Interaction Energies & Average $(\mathrm{kJ} / \mathrm{mol})$ \\
\hline CAT & $\mathrm{Pa}^{*}$ & Coulomb & $-219.9200 \pm 8.2$
\end{tabular}

Lennard-Jones $\quad-70.9474 \pm 5.8$

Total $-290.8674 \pm 10.0$

Coulomb $-107.1020=32.0$

Lennard-Jones $-82.0733 \pm 6.6$

Total $-189.1753 \pm 32.7$

EPL $\quad \mathrm{Pa}^{*} \quad$ Coulomb $-41.2606 \pm 3.6$

Lennard-Jones $-101.537 \pm 2.1$

Total $-142.7976 \pm 4.2$

$\mathrm{Sa}^{*} \quad$ Coulomb $-40.1841 \pm 1.5$

Lennard-Jones $\quad-114.11 \pm 7.5$

Total $-154.2491 \pm 7.6$

ORI $\quad \mathrm{Pa}^{*} \quad$ Coulomb $-244.537 \pm 10.0$

Lennard-Jones $-99.0065 \pm 1.5$

Total $-343.5435 \pm 10.1$

$\mathrm{Sa}^{*}$

Coulomb $-143.712=8.6$

Lennard-Jones $\quad-103.7 \pm 3.5$

Total $-247.412 \pm 9.3$

Note. Total interaction energies were calculated by the summation of Coulomb and Lennard-Jones interaction energies, with propagation of error estimation.

\begin{tabular}{|c|c|c|c|c|}
\hline Ligand & Bacteria & & Hbonds & Active Residues \\
\hline \multirow[t]{4}{*}{ CAT } & $\mathrm{Pa}^{*}$ & Before MD & 7 & ASP 8, LYS 70, GLU 171, GLY 197, GLU 267, ASP 368 \\
\hline & & After MD & 5 & LYS 70, GLU 171, ASP 194, GLU 267 \\
\hline & $\mathrm{Sa}^{*}$ & Before MD & 6 & ASP 8, GLY 10, THR 12, ASN 13, THR 35, SER 313 \\
\hline & & After MD & 1 & CYS 15 \\
\hline \multirow[t]{4}{*}{ EPL } & $\mathrm{Pa}^{*}$ & Before MD & 1 & ARG 345 \\
\hline & & After MD & 1 & ARG 345 \\
\hline & $\mathrm{Sa}^{*}$ & Before MD & 2 & ASN 13, LYS 240 \\
\hline & & After MD & 1 & THR 12 \\
\hline \multirow[t]{4}{*}{ ORI } & $\mathrm{Pa}^{*}$ & Before MD & 6 & THR 11, LYS 70, GLU 171, ASP 194, GLY 197, GLY 198 \\
\hline & & After MD & 9 & THR 11, THR 12, ASP 194, LEU 195, ASP 233, GLN 343, THR 344 \\
\hline & $\mathrm{Sa}^{*}$ & Before MD & 5 & THR 12, ASN 13, GLY 312, GLU 237, ASP 338 \\
\hline & & After MD & 2 & GLY 312, GLU 339 \\
\hline
\end{tabular}

Note. Hbonds refers to number of hydrogen bonds between receptor and ligand. Before MD refers to systems that went through energy minimization and NVT/NPT equilibration.

Figure 3. MD simulation of (A) P. aeruginosa and (B) S. aureus DnaK (brown)-Catechin(blue) complexes, of which P2Rank predicted druggable pocket residues were colored in green for better visualization. (C) Tabulated interaction energy values of DnaK-Catechin complexes. (D) Tabulated changes in number of hydrogen bonds and active residues before and after $\mathrm{MD}$. Note $\mathrm{B}^{\star}-\mathrm{Before} \mathrm{MD}, \mathrm{A}^{*}-\mathrm{After} \mathrm{MD}, \mathrm{Pa}^{*}-P$. aeruginosa, $\mathrm{Sa}^{*}-\mathrm{S}$. aureus, CAT-Catechin, EPL-Eplerenone, ORI-Oritin-4-beta-ol. All data were generated using GROMACS ${ }^{34}$ in-built functions.

mol, respectively for $S$. aureus $(\mathrm{S} a)$ and $P$. aeruginosa $(\mathrm{Pa})$ DnaK proteins. C, E, O also exhibited good predicted pharmacological properties except that $\mathrm{C}$ is a PGP substrate (Fig. 1, Table S5). The binding conformations of C (Fig. 2B), E (Fig. 2C), and O (Fig. 2D) to both DnaK proteins of $S a(\mathrm{SaD})$ and $\mathrm{Pa}(\mathrm{PaD})$ showed potential structural competitive inhibition of ATP binding at the docking pocket. Moreover, we observed rich electrostatic interactions (Fig. 2E) in $\mathrm{C}$ and $\mathrm{O}$, but not in $\mathrm{E}$, having only one intermolecular hydrogen bond.

Validation of inhibitory effects of selected compounds by Molecular Dynamics Simulation. To this end, we carried out Molecular Dynamics (MD) simulations for $10 \mathrm{~ns}$ for $\mathrm{C}$, E, and O Ligand-SaD/PaD complexes to observe ligand-receptor interactions. Throughout MD simulations, the ligands were retained in the docking pocket of respective $\mathrm{DnaK}$ receptors, except for $\mathrm{C}$ in the $\mathrm{SaD}$ system $(\mathrm{CSaD})$ of which the ligand seemed to be escaping from the initial binding pocket (Fig. 3A, B). Moreover, the upper part of the DnaK NBD domain was completely disintegrated in CSaD. Besides, the total number of receptor-ligand intermolecular hydrogen bonds were maintained stably at around 4 and 5 in P. aeruginosa DnaK complexed with $\mathrm{C}(\mathrm{CPaD})$ and $\mathrm{O}(\mathrm{OPaD})$ respectively, and 4 in S. aureus DnaK complexed with O (OSaD) (Fig. 4A). Moreover, both the E complexes of $\mathrm{SaD}(\mathrm{ESaD})$ and $\mathrm{PaD}(\mathrm{EPaD})$ have maintained the total number of hydrogen bonds at around 1 . However, in $\mathrm{CSaD}$, we observed a sharp decline in the number of intermolecular hydrogen bonds at the $5 \mathrm{~ns}$ time point from around 4 to between 0 and 1, which can explain the escape of ligand from its initial docking pocket. We also observed stable active residues in CPaD (LYS 70, GLU 171, GLU 267), EPaD (ARG 345), and OPaD (THR 11, ASP 194) complexes, as well as in OSaD (GLY 312) complex (Fig. 3D).

In all MD simulation systems, we found that the root-mean-square fluctuations of the DnaK receptor maintained at around $0.5 \mathrm{~nm}(5 \AA)$ except for the C-terminal end where the disordered regions were localized 

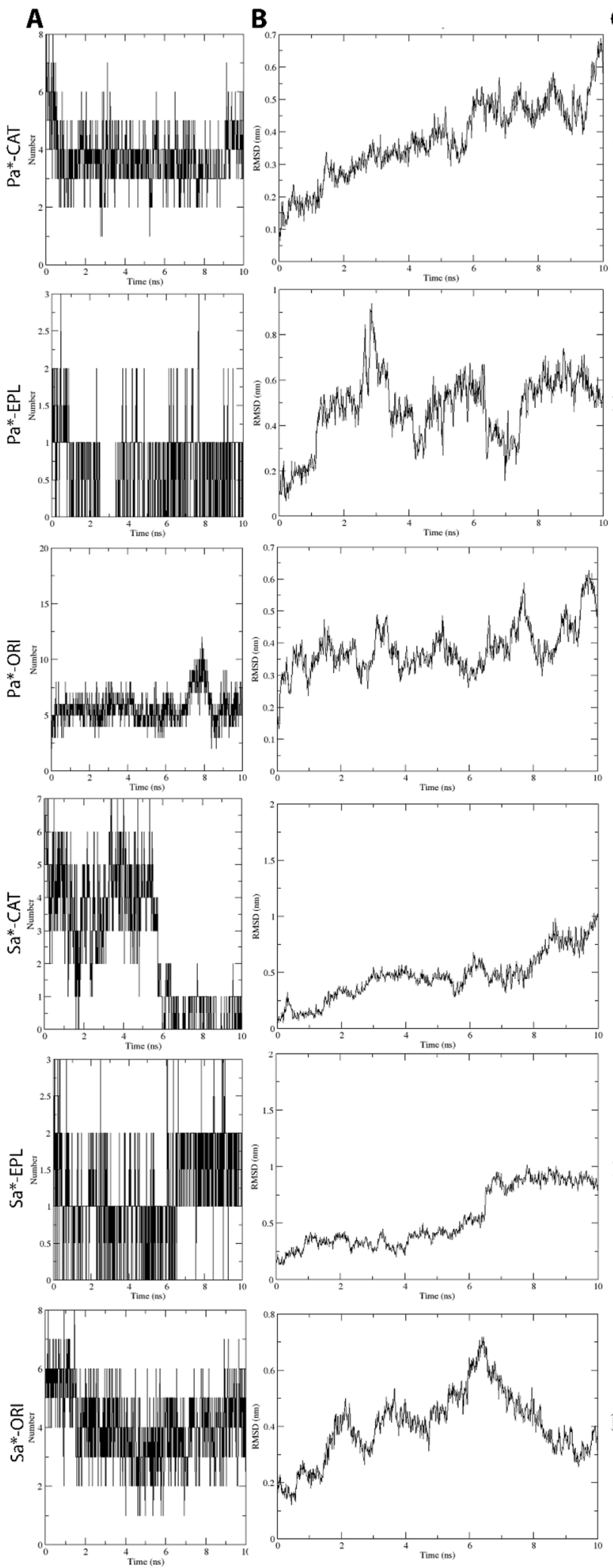

B
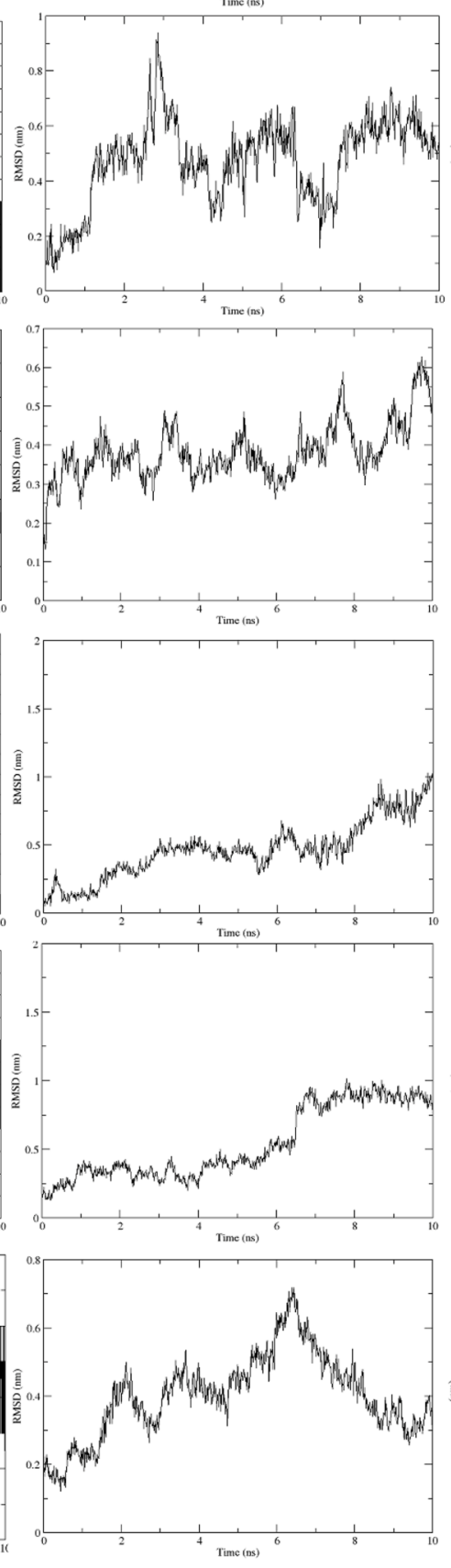

C
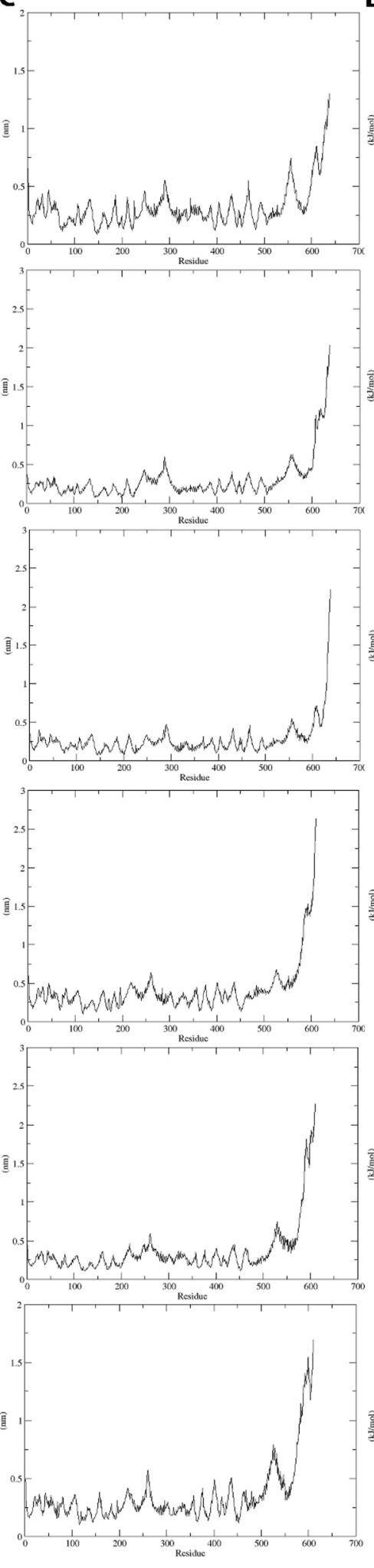

D
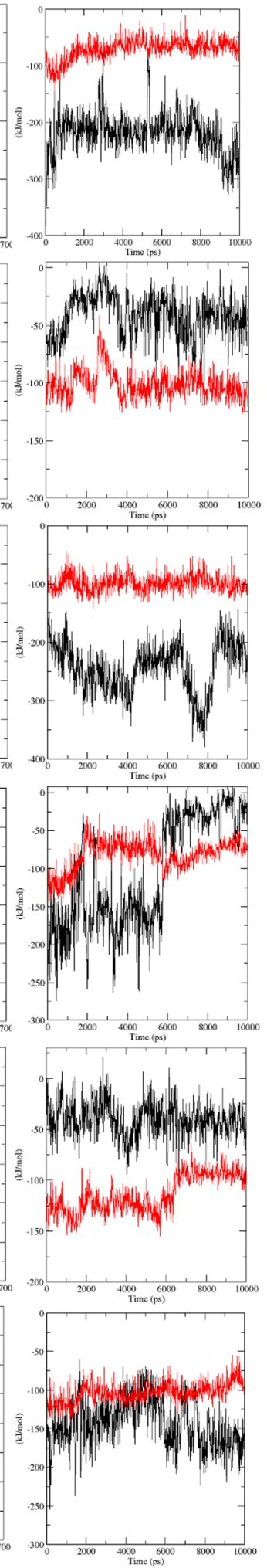

Figure 4. Receptor-ligand interactions over the course of MD simulation, of which (A) number of hydrogen bonds between receptor and ligand, (B) RMSD values of Catechin, (C) RMS fluctuation-per-residue of receptor macromolecule, and (D) receptor-ligand interaction energies (Black: Coulombic interaction energies, Red: Lennard-Jones energies) were computed over the course of $\mathrm{MD}$. Note $\mathrm{Pa}^{*}-P$. aeruginosa, $\mathrm{Sa}^{*}-\mathrm{S}$. aureus, CAT-Catechin, EPL-Eplerenone, ORI-Oritin-4-beta-ol. All data were generated using GROMACS $^{34}$ in-built functions. 
(Fig. 4C). Besides, we observed that the RMSD of $\mathrm{C}$ and $\mathrm{E}$ were maintained at $0.5 \mathrm{~nm}$ in the $\mathrm{PaD}$ receptor, as well as higher RMSD values of around $0.8 \mathrm{~nm}$ in CSaD and ESaD (Fig. 4B). The ligand RMSD were relatively lower in $\mathrm{O}$, compared to others, which was $0.4 \mathrm{~nm}$ in both $\mathrm{SaD}$ and $\mathrm{PaD}$ cases. The interaction energies of all systems maintained stably throughout the simulation, except for the CSaD complex of which a sharp decrease of Coulomb potential can be observed at around $6 \mathrm{~ns}$ time point (Fig. 4D). In general, E maintained the lowest total interaction energies, followed by $\mathrm{C}$ and $\mathrm{O}$ (Fig. 3C).

\section{Discussion}

Over the years, the commendable development in the field of virtual screening has enabled time- and costefficient drug discovery along with repurposing ${ }^{35}$. Herein, we have carried out a scaffolded approach to antimicrobial drug discovery from a yellow variety of Malaysian N. lappaceum L. fruit epicarp crude extracts. The first upstream set of experimental work comprised the extraction of the plant product, followed by characterization of their antimicrobial property and chromatographic identification of chemical compounds from therein. This was coupled with a downstream set of computational analyses comprising virtual screening and pharmacological predictions of extracted chemical compounds against potential drug targets. To this end, molecular dynamic simulation has taken a step forward to uncover new potent bioactive compounds which can target both gramnegative and -positive bacteria at the same time. Our study delineates a method to uncover potent chemicals which might have contributed to the antibacterial activities of plant products like Nephelium lappaceum epicarp, to be further utilized for drug discovery, repurposing, or other ab initio synthetic enhancements.

Extraction is the key stage to obtain the diverse bioactive chemical compounds from plant products. These chemical determinants display different solubility with different organic solvents such that screening with different solvents helps to bring forth the best one for further exploration ${ }^{36}$. Thus, we have utilized several organic solvents to explore the extraction of biologically active constituents. Herein, we initiated a sequential extraction process of utilizing solvents like chloroform (CF), ethyl acetate (EA), acetone (AC), ethanol (ET), methanol (MT) and water (WT), in order of their increasing polarity. Our study revealed that the yellow variety of Malaysian $N$. lappaceum epicarp crude extracts exhibited varied inhibitory activities against the six tested MDR pathogens, namely, B. subtilis, methicillin-resistant $S$. aureus (MRSA), S. pyogenes, P. aeruginosa, K. pneumoniae and $S$. enterica. Essentially, the $\mathrm{EA}(\mathrm{S})$ and $\mathrm{AC}(\mathrm{S})$ fractions notably inhibited the Gram-positive $S$. pyogenes and MRSA and the Gram-negative $P$. aeruginosa while the remaining solvent fractions responded moderately or poorly. This provided a strong clue for us to proceed for further direct extraction from EA(S) and AC(S) crude extract fractions (CEF). Thereafter, following the chromatographic analyses of HPLC, LC-MS and GC-MS of these $\mathrm{EA}(\mathrm{S})$ and $\mathrm{AC}(\mathrm{S}) \mathrm{CEF}$, we obtained different results from the direct extract fractions of EA and AC. Interestingly, we found the CEF of EA(D) and AC(D) to be more efficient via broth dilution than the disc diffusion methods (Tables 1 and 2). This could be attributed to the following fact. The different constituents of the CEF need to diffuse slowly in agar from a liquid to solid interphase in the agar diffusion method compared to the complete liquid interphase for broth microdilution.

It is important to note that despite similar reports to our findings by Mohamed et al. ${ }^{37}$; Thitilertdecha et al. ${ }^{38}$ and Tadtong et al. ${ }^{39}$, a comprehensive chemical profiling to unearth plausible determinants, potential enough against the MDR pathogens, is lacking to date. Thus, based on the prominent antibacterial effects of the $\mathrm{EA}(\mathrm{S})$ and $\mathrm{AC}(\mathrm{S})$ extracts of $N$. lappaceum fruit epicarp, we perceived these two fractions to harbor important bioactive molecules. Therefore, we subjected the sequential extracts of EA(S) and AC(S) fractions to HPLC analysis. The results confirmed the presence of some standard phenolic compounds with antioxidant properties namely, malic acid, vitamin $\mathrm{C}$, chlorogenic acid, epigallocatechin gallate, quercetin and catechin hydrate (Table 3). Notably, we found ethyl acetate and acetone as the competent solvents to extract total flavonoid and phenolic compounds ${ }^{36}$. Nazir et al. ${ }^{40,41}$, however, reported the afore-mentioned compounds in various other organic solvent extracts of Silybum marianum and Elaeagnus umbellate.

To this end, an extensive spectrum of chemical classes was revealed after LC-MS analysis and included, terpenes, alkaloids, polyunsaturated and monounsaturated fatty acids among others, that were present in both the extracts. Among these, only 21 of the 54 compounds (with above 86\% MFG scores) of the EA(S) fractions have been reported to possess antibacterial activities (Table S3). Interestingly, most of them have been newly reported (within the last five years) including $\mathrm{L} 2^{42}, \mathrm{~L} 3^{43}, \mathrm{~L} 6{ }^{44}, \mathrm{~L} 10^{46}, \mathrm{~L} 12^{47}, \mathrm{~L} 16^{48}, \mathrm{~L} 20^{49}, \mathrm{~L} 22^{51}, \mathrm{~L} 26^{52}, \mathrm{~L} 28^{53}$, $\mathrm{L} 31^{54}, \mathrm{~L} 46^{58}, \mathrm{~L} 48^{59}, \mathrm{~L} 51^{60}, \mathrm{~L} 53^{61}$ and L5 $4^{59}$. Others are known for some time, namely, L7 $7^{45}, \mathrm{~L} 21^{50}, \mathrm{~L} 35^{55}, \mathrm{~L} 37^{56}$ and L45 ${ }^{57}$. Similarly, the AC(S) extract fractions contained only 10 from 44 compounds with reported antibacterial activities (Table S4). Of these, except for $\mathrm{L} 24^{55}$ and $\mathrm{L} 35^{58}$, all were reported recently. These included L2 ${ }^{44}, \mathrm{~L} 5^{62}$, $\mathrm{L} 10^{48}, \mathrm{~L} 13^{52}, \mathrm{~L} 19^{54}, \mathrm{~L} 36^{58}, \mathrm{~L} 38^{59}$ and L42 ${ }^{61}$. Thus, possibilities exist for those 35 and 26 compounds from EA(S) and AC(S) fractions, respectively, with no matched identity with the library (Table S6 \& S7) to be medically important, though, further characterization is required to evaluate their usages.

We have authenticated a further revelation of important biomolecules through GC-MS (Table 4) besides the HPLC and LC-MS analyses mentioned above in Tables 3 \& S3-S4 respectively. Notably, we analyzed both the sequential and direct extracts of EA and AC fractions through GC-MS. Unlike the LC-MS reported compounds, however, about $50 \%$ of the chemical components, unearthed through GC-MS, are unknown for their antibacterial activity. For instance, in the case of sequential extracts, for both the EA(S) and AC(S) fractions, only 4 molecules were detected (with area \% scores above $0.5 \%$ ) without any a priori antibacterial activity (Table 4 ). Likewise, for the direct extract fractions of EA(D), only 5 out of the 8 compounds detected (with area \% scores above $0.5 \%$ ) were known to possess such activity. These are DGEA $1^{19}$, DGEA2 ${ }^{20}$, DGEA3 ${ }^{21}, \mathrm{DGEA} 4^{22}$ and DGEA $5^{23}$. However, for the AC(D) extract fraction, 4 out of the total 6 compounds detected, were reported to possess the antibacterial effect. These are DGAC2 ${ }^{19}, \mathrm{DGAC}^{24}, \mathrm{DGAC}^{20}$ and DGAC6 ${ }^{21}$. 
With a set of 91 compounds obtained through chromatographic analyses, we have conducted a computational analysis for virtual screening through molecular docking to shortlist a selective set of chemicals via pharmacokinetics consideration (Table S5). Pharmacokinetics is an important criterion when it comes to drug discovery and drug design, especially about bioavailability and toxicity. Herein, we have considered several parameters for absorption, metabolism, drug-likeness, and bioavailability for selecting the ideal drug for potential pharmacological application in the future. For instance, good GI absorption can allow absorption into the bloodstream during oral consumption, while bad blood-brain barrier (BBB) permeability can avoid interruption to the central nervous system ${ }^{63}$. P-glycoprotein (PGP) substrates are being actively effluxed from the cells thereby resulting in low absorption into the blood circulation ${ }^{64,65}$. Besides these, cytochrome P450 enzymes are crucial in the metabolism of most clinical drugs. Hence, the inhibition of cytochrome P450 enzymes can lead to decreased drug metabolism and possibly, adverse health complications, due to drug-drug interaction upon co-prescription with other drugs $s^{66,67}$. Moreover, the drug-likeness rules, such as the Lipinski rule of five, work by predicting pharmacological behavior upon oral administration based on the chemical properties of potential drugs $^{25}$. Lastly, bioavailability takes consideration of both absorption and distribution of the drugs, of which the eventual presence in blood circulation upon oral consumption is evaluated.

DnaK protein belongs to the $70 \mathrm{kDa}$ heat shock protein (HSP70) family, which functions as a molecular chaperone, mediated by its ATPase activities ${ }^{68}$. DnaK protein has been reported to be central in mediating bacterial stress responses. Among these, DnaK mutants have manifested an increase in antimicrobial susceptibilities and a decrease in survivability in the host ${ }^{69-71}$. Moreover, our previous work on whole-genome analysis (WGA) of protein interaction network (PIN) reported that DnaK protein was crucial in mediating quorum sensing in multidrug-resistant Proteus mirabilis ${ }^{72}$. Furthermore, WGA analyses of PIN from MDR pathogens like P. aeruginosa, S. aureus, S. enterica, S. pneumoniae, P. mirabilis, Acinetobacter baumannii, Escherichia coli and Mycobacterium tuberculosis revealed DnaK to be among the top 10 crucial proteins indispensable for the cellular integrity of the bacteria ${ }^{73}$. Also, the ATP-binding pocket of the DnaK chaperone has been indicated to be druggable and shown promise to cope with MDR in both gram negatives and positives as observed from an unpublished work of the same group of researchers. Hence, DnaK protein has been selected for the in-silico study, herein, as a promising drug target for MDR bacteria by inhibiting its ATP binding pocket, which can result in its impairment of chaperone function.

Through our computational screening of the chemical libraries of the N. lappaceum L. fruit epicarp extractions, we have shortlisted Catechin (C), Eplerenone (E), and Oritin-4-beta-ol (O) as the promising antimicrobials in combating the MDR pathogens by dint of their capacity in targeting the DnaK protein and having good pharmacological profiles. Despite being a PGP substrate, $\mathrm{C}$ has manifested a strong binding affinity to DnaK and therefore, can result in effective DnaK functional inhibition with a small amount. Otherwise, PGP inhibitors like $\mathrm{C}$ can be co-prescribed easily as it has a good metabolic profile. Moreover, $\mathrm{C}$ has been well-characterized for its antibacterial activities and known for its ability to cause leakage of bacterial cellular contents along with increased intracellular reactive oxygen species production in both gram negatives and positives ${ }^{74,75}$. However, the biological targets of $\mathrm{C}$ have not been described. As DnaK protein is crucial in bacterial stress response, by inhibiting the DnaK chaperone function, the bacterial cellular and biomolecular integrity can be effected upon receiving environmental oxidative stress. Herein, we showed that in P. aeruginosa, C could bind stably to the ATP-binding pocket of DnaK throughout the MD simulation with 3 stable active residues (LYS 70, GLU 171, and GLU 267), while maintaining the ATP-bound conformation of the DnaK protein without the necessity for ATP binding (Figs. 2B, 3A). This reflected the inability of the ATP molecules to bind the CPaD (Catechin-bound DnaK protein of $P$. aeruginosa) as also a complete halting of the normal DnaK chaperone function via conformational changes ensuing ATP hydrolysis. However, C could not inhibit $\mathrm{SaD}$ (DnaK of S. aureus) the same way, due to its inability to maintain the integrity of the NBD domain and thereby escaping from the binding pocket. It is this binding pocket that allows subsequent binding of ATP molecules on DnaK to continue the chaperone function. On the contrary, herein, we present the discovery of two novel potential compounds, E and O, whose antibacterial activities have not been reported and/or described earlier. Notably, E has been widely utilized in cardiovascular implications and as diuretics ${ }^{52,76}$. O, however, has not been explored to confer any biological significance. Despite that, it is notable that the chemical structure of $\mathrm{O}$ is analogous to $\mathrm{C}$ (Fig. 1), with the sites of hydroxylation being slightly different.

Throughout the molecular dynamics simulation (MDS) processes, we can only observe 1 or 2 hydrogen bonds in $\mathrm{EPaD}$ and $\mathrm{ESaD}$, which suggested weak protein-ligand electrostatic interactions. This can be explained by the chemical structure of E, being crowded with carbonyls and ethers which are weak bases, and hydroxyl groups are lacking. The ligand, however, has been retained in the docking pocket throughout MDS. This probably suggests that hydrophobic (van der Waals) interactions were dominant in this case. This was reflected through the intermolecular interaction energies (Fig. 4D), of which the Lennard-Jones potentials were much higher than Coulomb potentials in Eplerenone-DnaK (ED) complexes, while the reverse was observed in for $\mathrm{C}$ and $\mathrm{O}$. Moreover, the binding conformation of $\mathrm{E}$ in $\mathrm{PaD}$ did not "cover-up" completely at the binding site of phosphate groups of the ATP for which further wet-lab confirmation is required. Furthermore, among the three ligands simulated, $\mathrm{O}$ manifested the best binding capabilities to both $\mathrm{PaD}$ and $\mathrm{SaD}$ with rich intermolecular electrostatic interactions and the highest total interaction energies. After MDS, the active residues THR 11 and ASP 194 were retained in $\mathrm{OPaD}$, while GLY 312 was retained in OSaD. Again, despite being structurally analogous to C, O manifested good predicted pharmacological properties in all the aspects considered. Therefore, with better binding capabilities to DnaK receptor and pharmacological properties, herein we report $\mathrm{O}$ to be a more potent antibacterial compound compared to the well-known $\mathrm{C}$, which is active against both the gram-positive and -negative bacteria.

In the end, E, the compound not reported earlier to exhibit antibacterial properties against the tested promising pathogens MRSA and P. aeruginosa, demands a separate focus. Importantly, E has been found in both the $\mathrm{EA}(\mathrm{S})$ and $\mathrm{AC}(\mathrm{S}) \mathrm{CEF}$. However, the EA(S) showed no activity compared to the AC(S) CEF. This might probably 
be attributed to the interference of other chemicals in that $\mathrm{EA}(\mathrm{S})$ which might not have been the case for $\mathrm{AC}(\mathrm{S})$, probably, facing no interference and thus, showing activities. Thus, E can be a probable candidate as projected through our in-silico studies comprising screening of pharmacological properties followed by molecular dynamics simulation.

\section{Conclusion}

Our findings reinstate the promising antibacterial effects, of the yellow variety of Malaysian Rambutan (N. lappaceum L.) fruit epicarp crude extracts, against selected Gram-positive and Gram-negative MDR pathogens. In this context, particularly ethyl acetate and acetone (sequential and direct) extracts demonstrated remarkable antibacterial effects toward at least MRSA and P. aeruginosa among the six tested MDR pathogens, while remaining fractions including, chloroform, ethanol, methanol and water did not exhibit such potential. Nevertheless, we present the epicarp of $N$. lappaceum as a novel source for antibacterial compounds projecting catechin, eplerenone and oritin-4-beta-ol with high potential for the development of pharmaceutically valuable future drugs. Further studies are mandatory to separate the specifically mentioned three compound(s) responsible for the desired effects and to develop our knowledge on the other unseen potentials in N. lappaceum.

\section{Materials and methods}

Solvents. For the preparation of crude extracts, we have used all solvents, of HPLC grades. In the order of increasing polarities, these were Chloroform (99.9\%, Sigma-Aldrich, LiChrosolv, Malaysia), Ethyl acetate, Acetone (99.5\% Chemiz, Malaysia), Ethanol, Methanol (99.8\%, ChemAR, Systerm, Malaysia) and double distilled Milli-Q Type 1 water (MilliporeMerck, Germany). For LC-MS and GC-MS studies, we have used the solvents of MS grades.

Plant product. We purchased the yellow variety fruits of $N$. lappaceum L. from the local marketplace, Bandar Sunway, Selangor, Malaysia. We prepared the Herbarium voucher and deposited at Sunway University, Selangor Darul Ehsan, Malaysia. Thereafter, for our research, we carefully observed the fruit characters of rambutan and selected the epicarp according to the Descriptor for Rambutan ${ }^{77}$.

Tested microorganisms.For our study, we obtained six clinical isolates from the Department of Biological Sciences, Sunway University, Malaysia. These were Streptococcus pyogenes (ATCC-49399), Bacillus subtilis (ATCC-11774), methicillin-resistant Staphylococcus aureus (MRSA) (MTCC-381123), Pseudomonas aeruginosa (ATCC-10145), Klebsiella pneumoniae (ATCC-700603) and Salmonella enterica (ATCC-14028). We tested all these six bacterial strains to be resistant to at least five of the ten antibiotics tested for their resistivity/sensitivity profile and thus, considered them to be multidrug-resistant (Table S8).

Preparation of crude extracts. We prepared the epicarp crude extracts following the method of Do et al. ${ }^{78}$ using the solvents mentioned earlier for the direct extracts. For the sequential method of extraction, we used the mentioned solvents in the order of their increasing polarity viz. chloroform $<$ ethyl acetate $<$ acetone $<$ ethanol $<$ methanol $<$ water. In both cases, essentially, we removed the peels of $N$. lappaceum from the fruit and washed thoroughly with running, followed by, distilled water to remove contaminants and thereafter dried using a freeze-dryer. We ground the dried peels into a fine powder using an electric grinder. To produce different fractions of crude extracts, we extracted $10 \mathrm{~g}$ of powder in $100 \mathrm{ml}$ of selected solvents. Thereafter, we mixed the solution thoroughly by using an incubator shaker (Yihder LM-530D Incubator Shaker, Taiwan) for $24 \mathrm{~h}$. To separate supernatant, we centrifuged the solution (Eppendorf $5810 \mathrm{R}$ Centrifuge, Germany) at $4000 \mathrm{rpm}$ for $10 \mathrm{~min}$ at $4{ }^{\circ} \mathrm{C}$ to eliminate the leftover fine sediments. Finally, we concentrated the solvent extracts using a Rotary evaporator, and further with a vacuum concentrator until a viscous extract was obtained. We stored all the extracts at $4{ }^{\circ} \mathrm{C}$ for future experiments.

Potential in-vitro antibacterial activities of yellow rambutan fruit epicarp extracts. Disc diffusion assay. We consistently swabbed the seed culture of the tested pathogen on an agar plate. Then, we separately impregnated sterilized blank paper discs with different crude extract fractions and placed them on the agar plate. We incubated the plates at $37^{\circ} \mathrm{C}$ for $16 \mathrm{~h}$. We noted the antibacterial activity by measuring the diameter of the inhibition zone. We used gentamicin $(10 \mu \mathrm{g} / \mathrm{disc})$ as positive control and kept DMSO $(<1 \%)$ as a negative control. We ensured that all the experiments had technical triplicates and we performed them twice to render two biological replicates.

Broth dilution assay. We used a broth micro-dilution method to evaluate the minimum inhibitory concentration (MIC) values of crude extracts using Clinical \& Laboratory Standards Institute (CLSI) procedures. Essentially, we added each extract $(5 \mu \mathrm{l})$ into the wells of a 96 well plate comprising $10^{5} \mathrm{CFU} / \mathrm{ml}$ bacterial cells. We incubated the 96 well plates at $37^{\circ} \mathrm{C}$ for $16 \mathrm{~h}$. We kept the final concentrations ranging from 250 to $2000 \mu \mathrm{g} /$ $\mathrm{ml}$. In each test, we included three controls comprising, gentamicin $10 \mu \mathrm{g} / \mathrm{ml}$ (as positive), DMSO $<1 \%$ final concentration (as solvent) and bacterial inoculum (as negative). We have taken the MIC value as the lowest concentration of the tested extract showing inhibitory effect against the pathogens, recorded via the Microplate reader (TECAN, Infinite-M200-PRO). We confirmed all tests, having technical triplicates, twice. We observed promising results for both the fractions of ethyl acetate and acetone extracts with which we carried out all chromatographic analyses. 
Statistical tests. In the present study, we performed all the tests in triplicates and expressed the data obtained as the mean \pm standard deviation (S.D). We determined the P values using the student's T-test, two-tailed distribution, $\left(^{*}\right)$ is $P \leq 0.05$. These have been reflected in Tables 1 and 2 and Figure S2.

Exploration of chemical constituents through chromatographic analyses. High-performance Liquid Chromatography (HPLC). We used the ethyl acetate and acetone extracts as samples for qualitative phytochemical screening via HPLC via the Agilent-1260 infinity system, according to the reported method of $\mathrm{Zeb}^{79}$. Briefly, we mixed one-gram sample extract in methanol and water $(1: 1 ; 20 \mathrm{~mL} ; \mathrm{v} / \mathrm{v})$ and heated at $70^{\circ} \mathrm{C}$ for $1 \mathrm{~h}$ in a water bath. We centrifuged this mixture at $4000 \mathrm{rpm}$ for $10 \mathrm{~min}$ and filtered $2 \mathrm{ml}$ of the supernatant into HPLC vials through Whatman filter paper. We performed the separation via the Agilent-Zorbax-Eclipse column (XDB-C18). Column gradients system comprised solvent B and C. Solvent B consisted of deionized water: methanol: acetic acid having a ratio of 180: 100: 20; v/v while solvent $C$ had deionized water: methanol: acetic acid in the ratio of 80: $900: 20$; v/v. We started the gradient system by solvent B for $100 \%, 85 \%, 50 \%$ and $30 \%$ at $0,5,20$ and $25 \mathrm{~min}$ followed by solvent C (100\%) from 30 to $40 \mathrm{~min}$. Elution occurred after $25 \mathrm{~min}$. We set the ultraviolet array detector (UVAD) at $280 \mathrm{~nm}$ for the antioxidants analysis and documented the chromatogram using retention times. We carried out the UV spectra of compounds and accessible standards along with quantification by taking the per cent peak area. We measured the quantity of the antioxidants by the formula:

$$
C x=\frac{A x \times C s\left(\frac{\mu \mathrm{g}}{\mathrm{ml}}\right) \times V(\mathrm{ml})}{A s \times \text { Sample }(\text { wt. in } \mathrm{g})}
$$

$C x=$ Sample concentration; $A s=$ Standard peak area; $A x=$ Sample peak area; $C s=$ Standard concentration $(0.09 \mu \mathrm{g} /$ $\mathrm{ml})$.

Liquid chromatography and mass spectrometry (LC-MS). We analyzed a mixture of standards and new metabolites found in the ethyl acetate and acetone fractions via LC-MS, exactly as per the method reported by Yap et al. ${ }^{80}$. To eradicate systematic errors, we used a reference solution with the two ions, with $\mathrm{m} / \mathrm{z}$ of 121.0508 and $92,266.0097$, being selected for mass calibration. Finally, we ran the mass spectra for the compounds present in ethyl acetate (EA) and acetone (AC) fractions against the database of NIST (National Institutes of Standard and Technology, Gaithersburg, MD, USA) Mass Spectral Search Program-2009 version 2 for the documentation of homologous compounds over Agilent Mass-Hunter Qualitative Analysis B.05.00 software.

Gas chromatography-mass spectrometry (GC-MS). We subjected the ethyl acetate and acetone fractions to gas chromatography-mass spectrometry (GC-MS) analysis, using Agilent technologies model 7890B GC System coupled with Pegasus HT High Throughput TOFMS (Leco Corp., MI, USA). We injected an aliquot of an extract of $1 \mathrm{ml}$ into the GC-MS apparatus. Next, we used Agilent J\&W HP-5MS (phenylmethyl siloxane, length $30 \mathrm{~m}$, Dia. $0.32 \mathrm{~mm}$, Film, $0.25 \mu \mathrm{m}$ ) analytic column to separate components under an inert atmosphere of helium $(1.5 \mathrm{~mL} / \mathrm{min})$. Other standardized parameters utilized during the process: oven temperature of $80{ }^{\circ} \mathrm{C}(2 \mathrm{~min})$ was increased to a temperature of $300^{\circ} \mathrm{C}$ at the rate of $3{ }^{\circ} \mathrm{C} / \mathrm{min}$, solvent delay time was $5 \mathrm{~min}$, inlet line temperature was $225^{\circ} \mathrm{C}$, and ion source temperature was $250^{\circ} \mathrm{C}$. Mass spectra were taken at $70 \mathrm{eV}$ and the acquisition mode-scan was 20-1000 amu while sixty-four (64) minutes was the GC run time. We achieved the interpretation of mass spectrum and the documentation of phytochemicals present in the fractions via the database of NIST libraries.

Virtual screening of chemical determinants from chromatographic analyses. In silico protein model generation. We have chosen $\mathrm{S}$. aureus $(\mathrm{Sa})$ and $\mathrm{P}$. aeruginosa $(\mathrm{Pa})$ as gram-positive and gram-negative bacterial representatives for computational analyses of DnaK protein binding. 3D structures of DnaK proteins, from the aforesaid species, were generated via homology modelling using MODELLER version $9.24^{81}$. DnaK has two conformations, namely, the open or ATP-bound and the closed or ADP-bound conformation ${ }^{65}$. Herein, we focused on the open conformation of DnaK, to identify potential competitive inhibitors of ATP to prevent the proper functioning of DnaK protein.

We have obtained the protein sequences of $\mathrm{Sa}$ and $\mathrm{Pa}$ DnaK from UniProtKB with accession IDs of Q2FXZ2 and A6VCL8 ${ }^{82}$, respectively. To search for suitable homology modelling templates, we utilized both NCBI BLASTp and the MODELLER in-built build_profile.py ${ }^{81,83}$. For $\mathrm{Pa} \mathrm{DnaK}(\mathrm{PaD})$, the templates were full-length ATP-bound E. coli (Ec) DnaK protein structures (PDB ID: 5NRO, Chain: A, Query Coverage (QC): 94\%, Percent Identity (PI): 79.50\%, Resolution (R): $3.25 \AA$ A PDB ID: 4JNE, Chain: A, QC: 94\%, PI: 78.80\%, R: $1.96 \AA$ 的 and PDB ID: 4B9Q, Chain: A, QC: 94\%, PI: 77.96\%, R: $2.40 \AA$ A). For $S a$ DnaK (SaD), besides the afore-mentioned $E c$ DnaK (EcD) models, we selected one additional template, from Geobacillus kaustophilus DnaK protein (PDB ID: 2V7Y, Chain: A), due to the high percentage of sequence identity expected as per the gram-positive character of S. aureus and G. kaustophilus. As this template structure was in the closed conformation and we were only interested in the open conformation, only the Nucleotide Binding Domain (NBD, residues 1 to 350 in template model) which does not differ much in both conformations, were taken into consideration for homology modelling, and the remaining C-terminal residues modelling were guided by the $E c$ models to shape an open conformation. Therefore, the templates for SaD were (PDB ID: 2V7Y, Chain: A, Template Residues: 1-350, QC: 57\%, PI: 83.19\%, R: 2.37 Å; PDB ID: 5NRO, Chain: A, QC: 93\%, PI: 56.19\%, R: 3.25 Å; PDB ID: 4JNE, Chain: A, QC: $92 \%$, PI: 55.54\%, R: $1.96 \AA$ A and PDB ID: 4B9Q, Chain: A, QC: $94 \%$, PI: 55.43\%, R: $2.40 \AA$ ). The template sequences were aligned with the target sequence for homology modelling via the built-in function of MODELLER (Fig. S6A). 5 homology models were generated for each protein of $\mathrm{SaD}$ and $\mathrm{PaD}$, and the models with the 
lowest DOPE (discrete optimized protein energy) scores were selected for downstream virtual screening for both. We then validated the $\mathrm{SaD}$ and $\mathrm{PaD}$ homology models via Swiss-Model Structure Assessment and SAVES v5.0 servers $^{84}$ (Fig. S6).

Druggable pocket validation. To validate the druggability of the ATP docking pocket, we have conducted ligand binding site prediction using P2Rank from PrankWeb server ${ }^{85}$. P2Rank predicts the chemical druggability on protein solvent-accessible surface via a non-templated machine learning approach. The ATP binding pocket was predicted to be druggable and ranked first in both cases of $\mathrm{SaD}$ and $\mathrm{PaD}$ (Table S9; Fig. S7). Thus, we further considered these pockets from the $\mathrm{SaD}$ and $\mathrm{PaD}$ complexes to be targeted for virtual screening.

Molecular docking with chemical determinants. We utilized the POAP pipeline ${ }^{86}$ for an in silico virtual screening of the chemical compounds obtained through different chromatographic separation. We have obtained the SMILES notations of these compounds, and generated their 3D models (in mol2 format) through the POAP Ligand Preparation pipeline. To this end, we utilized Chimera to generate physiological protonation states of the ligands, and PDBQT files were prepared ${ }^{87}$. We also carried out ligand optimizations via the POAP Ligand Preparation pipeline utilizing the MMFF94 force field which is being optimized for drug-like organic molecules and molecular docking ${ }^{88}$. Out of the 50 conformers, generated for each ligand through the Weighted Rotor Search approach, only the best conformers were retained. Finally, we have subjected the ligands to energy minimization for 5000 steps by the conjugate algorithm.

We have prepared the macromolecule receptors, of the $\mathrm{SaD}$ and $\mathrm{PaD}$ proteins, using AutoDockTools. We utilized AutoDock 4.2, aided by the POAP pipeline, for the virtual screening process ${ }^{89}$. For AutoDock parameters, we have set 100 generations of Lamarckian Genetic Algorithm for each protein-ligand complex. To fit in the previously predicted pocket, we adjusted the docking grids into squares of $24 \AA$ with $\mathrm{x}, \mathrm{y}, \mathrm{z}$ coordinates of $17.647,75.43,27.766$, and $18.069,74.299,28.532$, for $\mathrm{SaD}$ and $\mathrm{PaD}$, respectively. For the silicon-containing compound among the set of ligands, we separately carried out molecular docking with AD4.1_bound parameter file, obtained from AutoDock, wherein we added the parameters for silicon atoms $(\mathrm{Rii}=4.3 \text {; eii }=0.402)^{90}$.

We have validated the docking methodology via redocking of experimentally confirmed and deposited structures with the reference ligand (Fig. S8). Therein, we have retrieved the ATP-bound E. coli DnaK crystallized structures from PDB (PDB ID: 4B9Q, CHAIN ID: A; PDB ID: 4JNE, CHAIN ID: A). The molecular docking search grids were squares of $24 \AA$, in $\mathrm{x}, \mathrm{y}, \mathrm{z}$ coordinates of $108.958,73.922,100.622$, and 19.081, 76.887, 31.16, for 4B9Q and 4JNE respectively.

Pharmacological properties screening. Using SwissADME ${ }^{91}$, we have carried out predictions on the pharmacological properties, encompassing pharmacokinetics, drug-likeness, and molecular information, for each chemical compound.

Molecular dynamics simulation. Ensuing virtual and pharmacological screenings, we rationally selected potential drug candidates to undergo molecular dynamics (MD) simulation via GROMACS version $2019.3^{34}$. We have utilized the CHARMM36 force field of version July 2020, along with the TIP3P water model, for macromolecule processing ${ }^{92}$. We used Avogadro software for mol 2 format conversion and complete protonation (protonation of non-polar atoms $)^{93}$. We also used a Perl script, sort_mol2_bonds.pl, written by Justin Lemkul for bond order arrangements in ligand mol 2 files. Then, we generated the topologies of the ligand models through the CGenFF server, and utilized a python script (cgenff_charmm2gmx.py) to convert topologies for CHARMM to GROMACS $^{94}$. We carried out solvation in a dodecahedron box ranged $1.0 \AA$ from the protein-ligand complex. The system was then ionized to achieve electrostatic neutralization. Subsequently, we subjected the system to energy minimization via the steepest descent algorithm until convergence at a maximum force of less than $1000 \mathrm{~kJ} \mathrm{~mol}^{-1} \mathrm{~nm}^{-1}$ (Fig. S9). Herein, we have monitored the potential energy shifts of the systems.

We carried out equilibration of the systems via NVT and NPT ensembles for 50,000 steps (100 ps), with temperature, pressure, and density shifts being monitored therein. Subsequently, we have carried out the production MD simulations for 5,000,000 steps (10 ns) to observe protein-ligand interactions. We computed the RMSD (Root Mean Square Deviation) values of ligands and receptors, number of hydrogen bonds between ligands and receptors, and ligand-receptor interaction energies (Coulombic interaction energies and Lennard-Jones energies) throughout the MD simulations. We have also computed thetotal interaction energies, and estimated the errorsvia error propagation by addition.

Generation of graphical illustrations. We utilized Matplotlib, a python library, to tabulate binding energies of all screened compounds ${ }^{31}$. We generated all 3D structural images using UCSF ChimeraX ${ }^{32}$, and $2 \mathrm{D}$ chemical structures using MarvinSketch ${ }^{33}$. Finally, we retrieved the figures for MD simulation analyses from the GROMACS in-built functions ${ }^{34}$.

Received: 9 December 2020; Accepted: 10 June 2021

Published online: 05 July 2021 


\section{References}

1. Maheshwari, M., Ahmad, I. \& Althubiani, A. S. Multidrug resistance and transferability of blaCTX-M among extended-spectrum $\beta$-lactamase-producing enteric bacteria in biofilm. J. Glob. Antimicrob. Resist. 6, 142-149 (2016).

2. Dockrill, P. The 12 deadliest drug-resistant bacteria have officially been ranked. Science Alert. https://www.sciencealert.com/the12-deadliest-drug-resistant-bacteria-have-officially-been-ranked (2017).

3. Obeng-Nkrumah, N. et al. High levels of extended-spectrum beta-lactamases in a major teaching hospital in Ghana: the need for regular monitoring and evaluation of antibiotic resistance. Am. J. Trop. Med. Hygiene 89(5), 960-964 (2013).

4. Barbieri, R. et al. Phytochemicals for human disease: An update on plant-derived compounds antibacterial activity. Microbiol. Res. 196, 44-68 (2017).

5. Cheesman, M. J. et al. Developing new antimicrobial therapies: are synergistic combinations of plant extracts/compounds with conventional antibiotics the solution?. Pharmacogn. Rev. 11(22), 57 (2017).

6. Djeussi, D. E. et al. Antibacterial activities of selected edible plants extracts against multidrug-resistant Gram-negative bacteria. BMC Complement. Altern. Med. 13(1), 164 (2013).

7. Mandalari, G. et al. Antimicrobial activity of flavonoids extracted from bergamot (Citrus bergamia Risso) peel, a byproduct of the essential oil industry. J. Appl. Microbiol. 103(6), 2056-2064 (2007).

8. Katalinić, V. et al. Polyphenolic profile, antioxidant properties and antimicrobial activity of grape skin extracts of 14 Vitis vinifera varieties grown in Dalmatia (Croatia). Food Chem. 119(2), 715-723 (2010).

9. Vattem, D. A. et al. Antimicrobial activity against select food-borne pathogens by phenolic antioxidants enriched in cranberry pomace by solid-state bioprocessing using the food grade fungus Rhizopus oligosporus. Process Biochem. 39(12), 1939-1946 (2004).

10. Kanatt, S. R., Chander, R. \& Sharma, A. Antioxidant and antimicrobial activity of pomegranate peel extract improves the shelf life of chicken products. Int. J. Food Sci. Technol. 45(2), 216-222 (2010).

11. Oliveira, D. A. et al. Nanoencapsulation of passion fruit by-products extracts for enhanced antimicrobial activity. Food Bioprod. Process. 104, 137-146 (2017).

12. Nguyen, N. M. P. et al. In vitro antioxidant activity and phenolic profiles of tropical fruit by-products. Int. J. Food Sci. Technol. 54(4), 1169-1178 (2019).

13. Zhuang, Y. et al. Protective effects of rambutan (Nephelium lappaceum) peel phenolics on $\mathrm{H}_{2} \mathrm{O}_{2}$-induced oxidative damages in HepG2 cells and d-galactose-induced aging mice. Food Chem. Toxicol. 108, 554-562 (2017).

14. Suhendi, A. Potential activity of Rambutan (Nepheliumlappaceum L.) fruit peel extract as antidiabetic and antihypercholesterolemia (2015).

15. Malini, C. \& Maheshkumar, R. Evaluation of bioactive potential in rambutan fruit (Nephelium lappaceum) samples using pathogens. Glob. J. Eng. Appl. Sci. 3(3), 138-142 (2013).

16. Bhat, R. S. \& Al-daihan, S. Antimicrobial activity of Litchi chinensis and Nephelium lappaceum aqueous seed extracts against some pathogenic bacterial strains. J. King Saud Univ. Sci. 26(1), 79-82 (2014).

17. Thitilertdecha, N., Teerawutgulrag, A. \& Rakariyatham, N. Antioxidant and antibacterial activities of Nephelium lappaceum L. extracts. LWT-Food Sci. Technol. 41(10), 2029-2035 (2008)

18. Sekar, M. et al. Comparative evaluation of antimicrobial properties of red and yellow rambutan fruit peel extracts. Annu. Res. Rev. Biol. 15, 3869-3874 (2014).

19. Padmavathi, A. R., Abinaya, B. \& Pandian, S. K. Phenol, 2, 4-bis (1, 1-dimethylethyl) of marine bacterial origin inhibits quorum sensing mediated biofilm formation in the uropathogen Serratia marcescens. Biofouling 30(9), 1111-1122 (2014).

20. Gul, P. \& Bakht, J. Antimicrobial activity of turmeric extract and its potential use in food industry. J. Food Sci. Technol. 52(4), 2272-2279 (2015).

21. Lee, H.-S. Antimicrobial properties of turmeric (Curcuma longa L.) rhizome-derived ar-turmerone and curcumin. Food Sci. Biotechnol. 15(4), 559-563 (2006).

22. Yusuf, A. J. et al. Antimicrobial activity of stigmasterol from the stem bark of Neocarya macrophylla. J. Med. Plants Econ. Dev. 2(1), $1-5$ (2018)

23. de Dieu Tamokou, J. et al. Antioxidant and antimicrobial activities of ethyl acetate extract, fractions and compounds from stem bark of Albizia adianthifolia (Mimosoideae). BMC Complement. Altern. Med. 12(1), 99 (2012).

24. Tintino, S. R. et al. Action of cholecalciferol and alpha-tocopherol on Staphylococcus aureus efflux pumps. EXCLIJ. 15, 315 (2016).

25. Lipinski, C. A. Lead-and drug-like compounds: the rule-of-five revolution. Drug Discov. Today Technol. 1(4), 337-341 (2004).

26. Ghose, A. K., Viswanadhan, V. N. \& Wendoloski, J. J. A knowledge-based approach in designing combinatorial or medicinal chemistry libraries for drug discovery. 1. A qualitative and quantitative characterization of known drug databases. J. Comb. Chem. 1(1), 55-68 (1999).

27. Veber, D. F. et al. Molecular properties that influence the oral bioavailability of drug candidates. J. Med. Chem. 45(12), 2615-2623 (2002).

28. Egan, W. J., Merz, K. M. \& Baldwin, J. J. Prediction of drug absorption using multivariate statistics. J. Med. Chem. 43(21), 3867-3877 (2000).

29. Muegge, I., Heald, S. L. \& Brittelli, D. Simple selection criteria for drug-like chemical matter. J. Med. Chem. 44(12), 1841-1846 (2001).

30. Hann, M. M. \& Keserü, G. M. Finding the sweet spot: the role of nature and nurture in medicinal chemistry. Nat. Rev. Drug Discov. 11(5), 355-365 (2012).

31. Hunter, J. D. Matplotlib: a 2D graphics environment. IEEE Ann. Hist. Comput. 9(03), 90-95 (2007).

32. Goddard, T. D. et al. UCSF ChimeraX: Meeting modern challenges in visualization and analysis. Protein Sci. 27(1), 14-25 (2018).

33. MarvinSketch (version 20.16), 2020, ChemAxon. http://www.chemaxon.com/.

34. Abraham, M. J. et al. GROMACS: high performance molecular simulations through multi-level parallelism from laptops to supercomputers. SoftwareX 1, 19-25 (2015).

35. Abdella, M., Abdella, B. \& Lahiri, C. Rediscovering and repurposing natural microbial macromolecules through computational approaches. Microb. Nat. Macromol. 65, 373-400 (2020).

36. Bakari, S. et al. Proximate analysis, mineral composition, phytochemical contents, antioxidant and antimicrobial activities and GC-MS investigation of various solvent extracts of cactus cladode. Food Sci. Technol. 37(2), 286-293 (2017).

37. Mohamed, S., Hassan, Z. \& Hamid, N. A. Antimicrobial activity of some tropical fruit wastes (guava, starfruit, banana, papaya, passionfruit, langsat, duku, rambutan and rambai). Pertanika 17, 219-219 (1994).

38. Thitilertdecha, N. et al. Identification of major phenolic compounds from Nephelium lappaceum L. and their antioxidant activities. Molecules 15(3), 1453-1465 (2010).

39. Tadtong, S. et al. Antibacterial activities of rambutan peel extract. J. Health Res. 25(1), 35-37 (2011).

40. Nazir, N. et al. Phytochemical analysis, molecular docking and antiamnesic effects of methanolic extract of Silybum marianum (L.) Gaertn seeds in scopolamine induced memory impairment in mice. J. Ethnopharmacol. 210, 198-208 (2018).

41. Nazir, N. et al. Phytochemical analysis and antidiabetic potential of Elaeagnus umbellata (Thunb.) in streptozotocin-induced diabetic rats: pharmacological and computational approach. BMC Complement. Altern. Med. 18(1), 332 (2018).

42. Song, F. et al. Antibacterial effect of fosfomycin tromethamine on the bacteria inside urinary infection stones. Int. Urol. Nephrol. 65, 1-10 (2019). 
43. Adamczak, A., Ożarowski, M. \& Karpiński, T. M. Antibacterial activity of some flavonoids and organic acids widely distributed in plants. J. Clin. Med. 9(1), $109(2020)$.

44. Karuppiah, V. \& Thirunanasambandham, R. Quebrachitol from Rhizophora mucronata inhibits biofilm formation and virulence production in Staphylococcus epidermidis by impairment of initial attachment and intercellular adhesion. Arch. Microbiol. 98, 1-14 (2020).

45. Metwally, A. M. et al. Phytochemical investigation and antimicrobial activity of Psidium guajava L. leaves. Pharmacogn. Mag. 6(23), $212(2010)$.

46. Sledz, W. et al. Antibacterial activity of caffeine against plant pathogenic bacteria. Acta Biochim. Pol. 62(3), 605-612 (2015).

47. Yi, H. S. et al. Impact of a bacterial volatile 2, 3-butanediol on Bacillus subtilis rhizosphere robustness. Front. Microbiol. 7, 993 (2016).

48. De, R. et al. Antimicrobial activity of ellagic acid against Helicobacter pylori isolates from India and during infections in mice. J. Antimicrob. Chemother. 73(6), 1595-1603 (2018).

49. Baspınar, Y., Kotmakçı, M. \& Öztürk, I. Antimicrobial activity of phytosphingosine nanoemulsions against bacteria and yeasts. Celal Bayar Üniversitesi Fen Bilimleri Dergisi 14(2), 223-228 (2018).

50. Kotsiou, A. et al. Impact of experimental trauma and niflumic acid administration on antimicrobials' concentration in serum and mandible of rats. J. Musculoskelet. Neuronal Interact. 6(3), 242 (2006).

51. Pham, T. H. et al. An update on the effects of glyceollins on human health: possible anticancer effects and underlying mechanisms. Nutrients 11(1), 79 (2019).

52. Pierson-Marchandise, M. et al. The drugs that mostly frequently induce acute kidney injury: a case-noncase study of a pharmacovigilance database. Br. J. Clin. Pharmacol. 83(6), 1341-1349 (2017).

53. da Silva Sa, F. A. et al. Phytochemical analysis and antimicrobial activity of Myrcia tomentosa (Aubl.) DC. Leaves. Molecules 22(7), 1100 (2017).

54. Heliawati, L., Syah, Y. M., \& Bumi, M. B. Bryononic acid: antibacterial compound from fruit hulls of S. koetjape Merr extract.

55. Rodríguez, P. et al. Estudio fitoquímico de hojas de Uncaria guianensis y evaluación de actividad antibacteriana. Acta Amaz 52, 303-310 (2011).

56. Bylka, W., Matlawska, I. \& Pilewski, N. A. Natural flavonoids as antimicrobial agents. JANA 7(2), 24-31 (2004).

57. Saradha Jyothi, K., \& Subba Rao, B. Scree IG of a tibacterial activity of Emblica officialis L. fruits.

58. Al-Harbi, R. et al. Antibacterial and anti-hemolytic activity of tannins from Pimenta dioica against methicillin resistant Staphylococcus aureus. Bangladesh J. Pharmacol. 12(1), 63-68 (2017).

59. $\mathrm{Vu}, \mathrm{T}$. T. et al. Antibacterial activity of tannins isolated from Sapium baccatum extract and use for control of tomato bacterial wilt. PLoS ONE 12(7), e0181499 (2017).

60. Sonfack, G. et al. Saponin with antibacterial activity from the roots of Albizia adianthifolia. Nat. Product Res. 54, 1-9 (2019).

61. Phuong, N. N. M. et al. Evaluation of antimicrobial activity of rambutan (Nephelium lappaceum L.) peel extracts. Int. J. Food Microbiol. 321, 108539 (2020).

62. Hinds, L. et al. Evaluating the antibacterial properties of polyacetylene and glucosinolate compounds with further identification of their presence within various carrot (Daucus carota) and Broccoli (Brassica oleracea) cultivars using high-performance liquid chromatography with a diode array detector and ultra-performance liquid chromatography-tandem mass spectrometry analyses. J. Agric. Food Chem. 65(33), 7186-7191 (2017).

63. Löbenberg, R. et al. Mechanism of Gastrointestinal Drug Absorption and Application in Therapeutic Drug Delivery 8-22 (Future Science Ltd, 2013).

64. Amin, M. L. P-glycoprotein inhibition for optimal drug delivery. Drug Target Insights 7, DTI-S12519 (2013).

65. Prachayasittikul, V. \& Prachayasittikul, V. P-glycoprotein transporter in drug development. EXCLI J. 15, 113 (2016).

66. Gong, Y. et al. Pharmacokinetics and pharmacodynamics of cytochrome P450 inhibitors for HIV treatment. Expert Opin. Drug Metab. Toxicol. 15(5), 417-427 (2019).

67. Granfors, M. T. et al. Differential inhibition of cytochrome P450 3A4, 3A5 and 3A7 by five human immunodeficiency virus (HIV) protease inhibitors in vitro. Basic Clin. Pharmacol. Toxicol. 98(1), 79-85 (2006).

68. Chiappori, F. et al. DnaK as antibiotic target: hot spot residues analysis for differential inhibition of the bacterial protein in comparison with the human HSP70. PLoS ONE 10(4), e0124563 (2015).

69. Singh, V. K. et al. Role for dnaK locus in tolerance of multiple stresses in Staphylococcus aureus. Microbiology 153(9), 3162-3173 (2007).

70. Yamaguchi, Y. et al. Effects of disruption of heat shock genes on susceptibility of Escherichia coli to fluoroquinolones. BMC Microbiol. 3(1), 1-8 (2003).

71. Wolska, K. I. et al. Antibiotic susceptibility of Escherichia coli dnaK and dnaJ mutants. Microb. Drug Resist. 6(2), 119-126 (2000).

72. Pawar, S. et al. In silico identification of the indispensable quorum sensing proteins of multidrug resistant Proteus mirabilis. Front. Cell. Infect. Microbiol. 8, 269 (2018).

73. Mujawar, S., El-Aal, A. A. A. A. \& Lahiri, C. Variant analysis from bacterial isolates affirms DnaK crucial for multidrug resistance. In International Work-Conference on Bioinformatics and Biomedical Engineering (Springer, Cham, 2020).

74. Ajiboye, T. O. et al. Contribution of reactive oxygen species to (+)-catechin-mediated bacterial lethality. Chem. Biol. Interact. 258, 276-287 (2016).

75. Sinsinwar, S. \& Vadivel, V. Catechin isolated from cashew nut shell exhibits antibacterial activity against clinical isolates of MRSA through ROS-mediated oxidative stress. Appl. Microbiol. Biotechnol. 104(19), 8279-8297 (2020).

76. Montalescot, G. et al. Early eplerenone treatment in patients with acute ST-elevation myocardial infarction without heart failure: the Randomized Double-Blind Reminder Study. Eur. Heart J. 35(34), 2295-2302 (2014).

77. IPGRI. 2003. Descriptors for Rambutan (Nephelium lappaceum). International Plant Genetic. Resources Institute, Rome, Italy. ISBN 92-9043-586.

78. Do, Q. D. et al. Effect of extraction solvent on total phenol content, total flavonoid content, and antioxidant activity of Limnophila aromatica. J. Food Drug Anal. 22(3), 296-302 (2014).

79. Zeb, A. A reversed phase HPLC-DAD method for the determination of phenolic compounds in plant leaves. Anal. Methods 7(18), 7753-7757 (2015).

80. Yap, W.-F. et al. Decoding antioxidant and antibacterial potentials of Malaysian green seaweeds: Caulerpa racemosa and Caulerpa lentillifera. Antibiotics 8(3), 152 (2019).

81. Webb, B. \& Sali, A. Comparative protein structure modeling using MODELLER. Curr. Protoc. Bioinform. 54(1), 5-6 (2016).

82. The UniProt Consortium. UniProt: the universal protein knowledgebase in 2021. Nucl. Acids Res. 2020;49(D1). https://www. google.com/url?q=https://www.uniprot.org/help/publications\&sa $=\mathrm{D} \&$ source=hangouts\&ust $=1615978252392000 \& u s g=\mathrm{AFQjC}$ NGsRzkhMdNS2yFdlQhkUjhXSnZSCw.

83. Altschul, S. F. et al. Gapped BLAST and PSI-BLAST: a new generation of protein database search programs. Nucleic Acids Res. 25(17), 3389-3402 (1997).

84. Waterhouse, A. et al. SWISS-MODEL: homology modelling of protein structures and complexes. Nucleic Acids Res. 46(W1), W296-W303 (2018)

85. Jendele, L. et al. PrankWeb: a web server for ligand binding site prediction and visualization. Nucleic Acids Res. 47(W1), W345W349 (2019). 
86. Samdani, A. \& Vetrivel, U. POAP: A GNU parallel based multithreaded pipeline of open babel and AutoDock suite for boosted high throughput virtual screening. Comput. Biol. Chem. 74, 39-48 (2018).

87. Pettersen, E. F. et al. UCSF Chimera-a visualization system for exploratory research and analysis. J. Comput. Chem. 25(13), 1605-1612 (2004).

88. Halgren, T. A. MMFF VI. MMFF94s option for energy minimization studies. J. Comput. Chem. 20(7), 720-729 (1999).

89. Morris, G. M. et al. AutoDock4 and AutoDockTools4: Automated docking with selective receptor flexibility. J. Comput. Chem. 30(16), 2785-2791 (2009).

90. Saito, H. et al. Phosphine boranes as less hydrophobic building blocks than alkanes and silanes: structure-property relationship and estrogen-receptor-modulating potency of 4-phosphinophenol derivatives. Bioorg. Med. Chem. 28(4), 115310 (2020).

91. Daina, A., Michielin, O. \& Zoete, V. SwissADME: a free web tool to evaluate pharmacokinetics, drug-likeness and medicinal chemistry friendliness of small molecules. Sci. Rep. 7, 42717 (2017).

92. Gutiérrez, I. S. et al. Parametrization of halogen bonds in the CHARMM general force field: improved treatment of ligand-protein interactions. Bioorg. Med. Chem. 24(20), 4812-4825 (2016).

93. Hanwell, M. D. et al. Avogadro: an advanced semantic chemical editor, visualization, and analysis platform. J. Cheminform. 4(1), 17 (2012).

94. Vanommeslaeghe, K. et al. Automation of the CHARMM general force field for drug-like molecules. Biophys. J. 100(3), 611a (2011).

95. Hall, T. A. BioEdit: a user-friendly biological sequence alignment editor and analysis program for Windows $95 / 98 / \mathrm{NT}$. Nucleic Acids Symp. Ser. 41(41), c1979-c2000 (1999).

\section{Acknowledgements}

The authors wish to acknowledge Sunway University, Selangor, Malaysia for the provision of necessary computational facilities. We thank Dr. Jactty Chew, Pang Jun Rui, Noor Akbar, Liew Tuck Onn and Rohit Mishra for providing assistance of varying kinds in different parts of the project.

\section{Author contributions}

C.L. conceived the concepts, planned and designed the analyses. A.A. extracted the natural products and assessed their antibacterial activities. M.Z. conducted and analyzed HPLC, helped by A.A. S.A. conducted LC-MS with the results being analyzed by A.A. and C.L. Y.Y.Y. provided the plant products and co-supervisory inputs along with E.K. Y.C.T. conducted the computational part with occasional inputs from C.L. Concepts for the artwork were generated and executed by A.A. and Y.C.T. with guidance provided by C.L. A.A. and Y.C.T. primarily wrote the manuscript aided by editorial up-gradation by C.L.

\section{Competing interests}

The authors declare no competing interests.

Additional information

Supplementary Information The online version contains supplementary material available at https://doi.org/ $10.1038 / \mathrm{s} 41598-021-92622-0$.

Correspondence and requests for materials should be addressed to C.L.

Reprints and permissions information is available at www.nature.com/reprints.

Publisher's note Springer Nature remains neutral with regard to jurisdictional claims in published maps and institutional affiliations.

(c) (1) Open Access This article is licensed under a Creative Commons Attribution 4.0 International (c) License, which permits use, sharing, adaptation, distribution and reproduction in any medium or format, as long as you give appropriate credit to the original author(s) and the source, provide a link to the Creative Commons licence, and indicate if changes were made. The images or other third party material in this article are included in the article's Creative Commons licence, unless indicated otherwise in a credit line to the material. If material is not included in the article's Creative Commons licence and your intended use is not permitted by statutory regulation or exceeds the permitted use, you will need to obtain permission directly from the copyright holder. To view a copy of this licence, visit http://creativecommons.org/licenses/by/4.0/.

(c) The Author(s) 2021 